\title{
Weather Based Yield Prediction and PDI Model for Grape Production Quality Forecast in Tamil Nadu using Mathematical Modelling
}

\author{
A. Eswari* \\ Department of Physical Sciences \& Information Technology, Agricultural Engineering \\ College \& Research Institute, Tamil Nadu Agricultural University, Coimbatore - 641003 , \\ Tamil Nadu, India \\ *Corresponding author
}

\section{A B S T R A C T}

Keywords

Primary and secondary infection,

Grape yield,

Climatic variables,

Mathematical

modelling, Disease

incidence;

Simulation

Article Info

Accepted:

18 March 2021

Available Online:

10 April 2021
In this paper, we developed the weather based yield prediction model between weather factors such as maximum temperature (Tmax), minimum temperature (Tmin), relative humidity (RH1), (RH2) and rainfall (RF) observed at Grapes Research station, Theni district during the summer and winter season from 2016 to 2020. This model is built using multiple regression analysis to explain the relationship between grape yield as well as climatic parameters and quality assessment. Our findings indicate that the models provide an effective way to the evaluate and forecast. Furthermore, we show that there exits many factors like downy mildew and powdery mildew to affect the quality of grape, our yield prediction model provide superior performance for grape analysis. Furthermore, the PDI model was developed to forecasting disease incidence; yield of grape by using climatic scenario. A comparison of our estimated results with the numerical simulation and experimental result available is provided.

\section{Introduction}

Mathematical models are at the heart of agricultural sciences and engineering and capture the essential aspects of systems, processes, phenomena. Models are founded on universally accepted laws of physics and chemistry. Also models are the key for understanding, predicting, designing, optimizing and controlling (Hans Peter Fischer et al., 2008).
Grapes vine originally a temperate fruit crop and it's also grown successfully under tropical conditions. A grape is one of the economically important fruit crops grown in India. The majority of the grapes produced in the country are used for table purpose and a small quantity is converted into raisin, wine and juice. Unripe grapes were used to treat sore throats, and dried grapes were used against constipation and thirst. Round, ripe, sweet grapes were used to treat wide range of health problems 
including cancer, cholera, smallpox, nausea, eye infections, skin, kidney, and liver diseases. But grapes, or the chemicals within them, especially oligomeric proanthocyanidin complexes have been touted as powerful antioxidants. There is good evidence that grapes seed extract can helps in treating chronic venous insufficiency and edema.

Cultivation of grapes in India has been commercially taken up presently under a wide range of soil and climate conditions in the states of Maharastra, Karnataka, Telungana, Andhra Pradesh and Tamil Nadu.

It is one of the most remunerative farming enterprises that, has created interest among Indian growers, which is evident in the steep increase in the area under grapes cultivation in the country during recent decades. Grape has a variety of pests and pathogens that cause economic damage (Bournier 1977, Flaherty et al., 1981, Ragusa et al., 2006) and that require control interventions.

Climate is one of the most important external factors determining the quality of fruits. Among climatic factors, temperature has a vital role on the quality as well as on the earliness of the crop. The weather parameters like viz., sun light, rainfall, humidity also significance influences quality development of the fruits.

Powdery mildew caused by Uncinulanecator (Braun and Takamatsu, 2000) is an important deadly disease of grapevine throughout the world. It is not possible to harvest good edible quality fruits without prophylactic measures.

It is prevalent in most grapevine growing region of the world and more serious than downy mildew in relatively dry areas. Powdery mildew infects all green tissues of the grapevine. On leaves, it appears as a white or grayish-white powdery covering of the upper and lower surfaces. Heavily infected leaves turn dull, dry out and drop prematurely.

Fruit infections may appear white and powdery or dark and dusty and sometimes result in shriveling or cracking of the berries (Wilcox, 2003).

Moderate to low relative humidity and low light favours the disease incidence. Absence of free moisture is essential since it inhibits germination of conida and may actually cause these spores to burst. The optimal temperature range the known the disease spread is 20$27^{\circ} \mathrm{C}$.

Vittorio Faluom et al., 2019used the source sink approach to integrated vine and grapevine mathematical model for vegetative development and production quality forecast.

Fengjiao fan et al., 2015 have analysed the grapewine quality evaluation using statistical model. Tanmay A. Wagh et al., 2019 has discussed the grapes leaf disease detection using Convolutional Neural Network. M. Bonfigli et al., 2020 performed the mathematical model here developed is based on first principles, in order to more accurately describe the underlying phenomena that govern the extraction process behavior. $\mathrm{T}$. Caffi and V. Rossi 2010applied simulation model to evaluation of a warning system for controlling primary infections of grapevine downy mildew.

Therefore, taking above facts into consideration, the present investigation has been undertaken to developing yield prediction and PDI model in grapes with respect to disease incidence, yield and climatic variables. These model are applied to use enhance or alter the natural growth and fruit development pattern or it can be used to control (or) prevent pest and disease outbreak in grapevines. 


\section{Determination of developing grape weather model and methods}

Data on weather parameters viz., maximum temperature (Tmax), minimum temperature (Tmin), rainfall (mm), relative humidity (RH1), (RH2) and Rainfall (RF) for 5 years (2016-2020) for south region of Tamil Nadu.

For model formulation, monthly weather data for five years (2016-2020) and yield prediction were used, in which forecast yield for grapes of different years was compared with monthly mean values of each of the weather parameters individually. Hence it was possible to identify the most important month with respect to each of the weather factors and use relevant values to develop a multiple linear weather based model using mathematical software. The model was validated through 5- year (2016-2020) independent dataset on weather, disease incidence and yield by statistical packages. Model performance was evaluated by comparing observed and predicted grape yield. The weather based yield prediction model is expressed in the following form:

Yield $=50.755+(-1.040) *$ Temperature +1.057

Yield $=19.012+(0.047) *$ Relative Humidity +1.224

Yield $=22.905+(-0.005) *$ Rain fall +1.206

The above three equations can be combined is as below

Yield=116.1435-2.6339*Temperature0.2696*Relative Humidity-0.0250*Rainfall

and, the percentage disease incidence for grape versus weather data is of the form

$\mathrm{PDI}=194.2031+(-3.7436) *$ Temperature + $(-$ $0.8952) *$ Relative Humidity+(-0.0059)* Rain fall +3.839

\section{Model validation}

Regression equation (4) was accepted as final model because equations (1), (2) and (3) included either all or fewer of RF, RH and Tmin and Tmax that were subsequently observed not to have influenced yield catches. The PDI model was validated using WASP 2.0 satisfactorily $(\mathrm{R}$ Square $=0.484$; Multiple Correlation Coefficient $(\mathrm{R})=0.696)$ with independent 5-year data from 2016-2020 on climatic parameters and yield. Besides, significance of ' $\mathrm{F}$ ' for these regression equations was standard error $=3.839$ for equation (5). The result of the regression for PDI by using WASP 2.0 can be described ANOVA table shown in the following table 1as:

Coefficient of determination $(\mathrm{R}$ Square $)=$ 0.484

Multiple Correlation Coefficient $(\mathrm{R})=0.696$ Standard Error $=3.839$

\section{Estimation of parameters}

From the equation (4) we can get the algebraic expression of climatic parameters of yield prediction model as follows:

$$
\begin{aligned}
& \text { Yield=167.0755+(-3.6157)*T+(-0.5043)*RH } \\
& +(-0.2623) * \mathrm{PDI} \\
& \text { (i.e.,) } \quad \text { Yield }=f(T, R H, R F, P D I)
\end{aligned}
$$

This is of the form $y=a \mathrm{X}_{1}+b \mathrm{X}_{2}+c \mathrm{X}_{3}+\mathrm{dX}_{4}$. Fitting a first degree regression curve using regression analysis, we can find Temperature $\left({ }^{0} \mathrm{C}\right)$, Relative humidity $(\%)$ and Rainfall $(\mathrm{mm})$ and PDI (\%) using the above equation (6).

\section{Numerical Simulation}

We present numerical simulations in this section to test the exactness and reliability of the proposed model. The result obtained in 
this paper will be compared with the highly accurate numerical solutions obtained by the MATLAB, OPSTAT and WASP 2.0, which implements the programme. The experimental and numerical values were plotted on the same coordinates for a wide range of possible values of the underlined problem parameters. Figure 2 that the derived analytical expressions for the Relative humidity, Temperature, Rain fall and yield are in strong agreement with numerical solution using MATLAB [10].The MATLAB used pdex4 function is also given in Appendix A. In this figure, the temperature and relative humidity increases then yield is also increases for the fixed value of PDI $=30-90 \%$ and rainfall is $20 \mathrm{~mm}$. the climatic parameters are inferences the yield and it increase up to certain level, thereafter it will decreased.

\section{Results and Discussion}

About the user of farmers of Theni district have a weather station on their one of the Grapes Research station. The weather forecast model of grapes uses hourly rainfall, temperature and relative humidity measurements to make the decisions about optimal weather conditions for downy and powdery mildew disease infection. Leaf wetness is now calculated from relative humidity and air temperature as sensors for leaf wetness proved inaccurate is plotted in figure 3 . For the primary infections period, the most critical conditions include receiving a 24-h cumulated rainfall of more than $10 \mathrm{~mm}$ and having leaves wet for 3 hours when the 24 -h average air temperature was $>10^{\circ} \mathrm{C}$. For secondary infection to be predicted, conditions must have included 4 continuous hours when relative humidity was $>92 \%$ with at least 2 or more having wet leaves as well as a mean air temperature $\geq 13^{\circ} \mathrm{C}$ during these hours are shown in figure 4. These favourable conditions are then marked on a diagram together with a graph of the weather conditions over the last 5 years. These climatic data from GRS, Theni are shown in table 2 . The disease incidence model also gives 4 risk classes $(0 \% ; 1-34 \% ; 35-74 \% \&>75 \%)$ for both primary and secondary infection by downy and powdery mildew diseases. Some producers would prefer a simpler report layout, while others understand and want weather data graphs (See figure 5).

Sum of active temperatures during different days of vegetation of grapesis plotted in figure 6. In this figure, the sum of active temperatures must comprise for all types of varieties more than $30-40^{\circ} \mathrm{C}$. At the sum of active temperature less than $20-27^{\circ} \mathrm{C}$, the vegetation of the grape culture in the south region of India is practically impossible. From figure 7 , it is observed that the value of relative humidity is more than $75 \%$. At this time unfavoured the powdery mildew incidence. From figure 8 , it is inferred that, the value of rainfall increases $250 \mathrm{~mm}$ as the function of calendar months (winter period). The disease incidence was assessed in and around Cumbum, Chinnamanur and Theni grape vineyards. Fifteen different grapes vineyards were selected for collection of disease incidence. The observations on disease incidence were collected twice a week as per the observation sheets in selected farmer's field. In the survey conducted for grape diseases incidence, Downy mildew (Plasmoparaviticola) was the major disease than other diseases. It was found to be major disease especially 0-60 days after forward pruning. The downy mildew incidence and intensity at field level during 2016- 2020 were assessed and presented in figure 9 and table 3 . Based on the survey, the highest incidence of downy mildew (28.02 PDI) was recorded during 2017-2018. The maturing bunches were also found to be affected by downy mildew in some orchards which affecting the quality of grapes. 
Table.1 Experimental parameters

\begin{tabular}{|c|c|c|c|c|c|}
\hline $\begin{array}{c}\text { Independent } \\
\text { Variables }\end{array}$ & Average & $\begin{array}{c}\text { Reg. } \\
\text { coefficients (b) }\end{array}$ & $\begin{array}{c}\text { Standard Error } \\
\text { (SE(b)) }\end{array}$ & T Test & T table (0.05) \\
\hline Temperature & 27.070 & -3.744 & 5.589 & -0.670 & $\mathbf{1 2 . 7 0 6}$ \\
\hline RH & 76.598 & -0.895 & 1.191 & -0.752 & $\mathbf{1 2 . 7 0 6}$ \\
\hline Rainfall & $\mathbf{6 4 . 7 4 2}$ & $\mathbf{- 0 . 0 0 6}$ & $\mathbf{0 . 0 6 4}$ & $\mathbf{- 0 . 0 9 1}$ & $\mathbf{1 2 . 7 0 6}$ \\
\hline
\end{tabular}

\begin{tabular}{|c|c|c|c|c|c|}
\hline \multicolumn{7}{|c|}{ ANOVAs Table } \\
\hline $\begin{array}{c}\text { Source of } \\
\text { variation }\end{array}$ & $\begin{array}{c}\text { Degrees of } \\
\text { freedom }\end{array}$ & $\begin{array}{c}\text { Sum of } \\
\text { squares }\end{array}$ & $\begin{array}{c}\text { Mean sum of } \\
\text { squares }\end{array}$ & F cal & F prob. \\
\hline Regression & 3 & 13.817 & 4.606 & 0.312 & $\mathbf{0 . 8 2 8}$ \\
\hline Residual & 1 & 14.740 & 14.740 & - & - \\
\hline Total & 4 & - & - & - & - \\
\hline
\end{tabular}

Intercept $(\mathrm{a})=194.203$

Table.2 Monthly meteorological data (2016-2020) from Theni district

\begin{tabular}{|c|c|c|c|c|}
\hline \multirow[t]{2}{*}{ Month } & \multicolumn{2}{|c|}{ Temperature $\left({ }^{0} \mathrm{C}\right)$} & \multirow{2}{*}{$\begin{array}{c}\text { Relative Humidity } \\
(\%)\end{array}$} & \multirow{2}{*}{$\begin{array}{c}\text { Rainfall } \\
\text { (mm) }\end{array}$} \\
\hline & Maximum & Minimum & & \\
\hline Feb 2016 & 31.20 & 20.64 & 82.00 & - \\
\hline Mar 2016 & 35.46 & 22.19 & 87.21 & 2.06 \\
\hline Apr 2016 & 38.14 & 25.13 & 71.44 & 3.15 \\
\hline May 2016 & 34.53 & 25.88 & 49.00 & 14.96 \\
\hline Jun 2016 & 30.20 & 24.04 & 73.86 & 1.58 \\
\hline Jul 2016 & 30.96 & 23.81 & 76.13 & 2.00 \\
\hline Aug 2016 & 31.16 & 24.91 & 74.87 & 1.01 \\
\hline Sep 2016 & 31.86 & 23.84 & 77.13 & 0.08 \\
\hline Oct 2016 & 3136 & 22.47 & 75.00 & 5.23 \\
\hline Nov 2016 & 31.14 & 21.76 & 80.10 & 0.53 \\
\hline Dec 2016 & 30.65 & 19.67 & 86.56 & 0.69 \\
\hline Jan 2017 & 30.30 & 26.80 & 88.22 & 43.00 \\
\hline Feb 2017 & 32.40 & 17.60 & 85.07 & 1.00 \\
\hline Mar 2017 & 32.90 & 21.40 & 81.45 & 77.00 \\
\hline Apr 2017 & 36.90 & 22.40 & 78.83 & 44.00 \\
\hline May 2017 & 34.80 & 23.30 & 74.64 & 97.00 \\
\hline Jun 2017 & 31.20 & 23.60 & 73.03 & 44.00 \\
\hline Jul 2017 & 31.70 & 24.30 & 69.67 & 28.00 \\
\hline Aug 2017 & 31.60 & 23.20 & 68.93 & 60.00 \\
\hline Sep 2017 & 30.70 & 22.50 & 68.03 & 214.00 \\
\hline Oct 2017 & 31.40 & 22.30 & 71.87 & 113.00 \\
\hline Nov 2017 & 30.00 & 21.20 & 75.80 & 233.00 \\
\hline Dec 2017 & 28.80 & 19.60 & 74.29 & 64.00 \\
\hline Jan 2018 & 30.60 & 16.80 & 76.83 & 2.00 \\
\hline Feb 2018 & 32.00 & 17.30 & 73.17 & 21.00 \\
\hline Mar 2018 & 33.19 & 21.32 & 76.77 & 22.00 \\
\hline
\end{tabular}




\begin{tabular}{|c|c|c|c|c|}
\hline Apr 2018 & 35.25 & 22.58 & 76.03 & 25.00 \\
\hline May 2018 & 33.19 & 23.09 & 79.09 & 181.00 \\
\hline Jun 2018 & 30.60 & 23.76 & 78.20 & 34.00 \\
\hline Jul 2018 & 29.22 & 22.70 & 76.48 & 118.50 \\
\hline Aug 2018 & 29.09 & 22.93 & 76.83 & 131.50 \\
\hline Sep 2018 & 32.33 & 22.33 & 75.23 & 142.00 \\
\hline Oct 2018 & 30.67 & 21.96 & 79.22 & 250.00 \\
\hline Nov 2018 & 30.93 & 22.03 & 78.63 & 137.00 \\
\hline Dec 2018 & 29.25 & 20.90 & 77.97 & 13.00 \\
\hline Jan 2019 & 28.51 & 16.80 & 79.70 & 0.00 \\
\hline Feb 2019 & 31.74 & 19.42 & 71.16 & 17.00 \\
\hline Mar 2019 & 35.14 & 20.64 & 76.29 & 32.00 \\
\hline Apr 2019 & 35.33 & 23.80 & 78.07 & 103.00 \\
\hline May 2019 & 36.35 & 26.51 & 78.96 & 121.00 \\
\hline Jun 2019 & 32.74 & 23.84 & 76.13 & 41.00 \\
\hline Jul 2019 & 31.22 & 22.90 & 78.41 & 65.00 \\
\hline Aug 2019 & 28.41 & 22.54 & 77.58 & 119.00 \\
\hline Sep 2019 & 29.76 & 22.76 & 77.00 & 168.00 \\
\hline Oct 2019 & 29.96 & 22.29 & 82.19 & 233.00 \\
\hline Nov 2019 & 32.23 & 27.16 & 81.10 & 112.00 \\
\hline Dec 2019 & 28.90 & 23.30 & 81.19 & 69.00 \\
\hline Jan 2020 & 28.54 & 17.09 & 77.70 & 0.00 \\
\hline Feb 2020 & 28.38 & 22.16 & 77.44 & 0.00 \\
\hline Mar 2020 & 33.70 & 20.90 & 76.38 & 0.00 \\
\hline Apr 2020 & 34.93 & 22.80 & 76.23 & 104.00 \\
\hline May 2020 & 33.77 & 23.42 & 77.61 & 37.00 \\
\hline Jun 2020 & 29.70 & 23.70 & 82.13 & 23.00 \\
\hline July 2020 & 29.29 & 26.61 & 81.32 & 140.00 \\
\hline
\end{tabular}


Table.3 Percentage disease incidence of downy mildew for grape during the year 2016-2020 from Theni district

\begin{tabular}{|c|c|c|c|c|c|c|c|c|c|c|c|c|}
\hline & & \multicolumn{10}{|c|}{ Raw Data format } & \\
\hline 2016-17 & 2016-17 & RI & & & & $\mathrm{R} 2$ & & & & R3 & & \\
\hline field no & $\begin{array}{l}\text { Per cent } \\
\text { disease } \\
\text { incidence }\end{array}$ & $\begin{array}{c}\text { No. of } \\
\text { grapevine } \\
\text { leaves } \\
\text { affected }\end{array}$ & $\begin{array}{c}\text { Total no. } \\
\text { of leaves } \\
\text { observed } \\
\text { in a set }\end{array}$ & $\begin{array}{c}\text { field } \\
\text { no }\end{array}$ & $\begin{array}{c}\text { Per cent } \\
\text { disease } \\
\text { incidence }\end{array}$ & $\begin{array}{c}\text { No. of } \\
\text { grapevine } \\
\text { leaves } \\
\text { affected }\end{array}$ & $\begin{array}{c}\text { Total no. of } \\
\text { leaves } \\
\text { observed in a } \\
\text { set }\end{array}$ & $\begin{array}{c}\text { field } \\
\text { no }\end{array}$ & $\begin{array}{c}\text { Per cent } \\
\text { disease } \\
\text { incidence }\end{array}$ & $\begin{array}{c}\text { No. of } \\
\text { grapevine } \\
\text { leaves } \\
\text { affected }\end{array}$ & $\begin{array}{c}\text { Total no. } \\
\text { of leaves } \\
\text { observed } \\
\text { in a set }\end{array}$ & $\begin{array}{c}\text { Mean } \\
\text { PDI }\end{array}$ \\
\hline 1 & 25.00 & 3 & 12 & 1 & 40.00 & 4 & 10 & 1 & 18.18 & 2 & 11 & 27.73 \\
\hline 2 & 18.18 & 2 & 11 & 2 & 8.33 & 1 & 12 & 2 & 16.67 & 2 & 12 & 14.39 \\
\hline 3 & 25.00 & 3 & 12 & 3 & 16.67 & 2 & 12 & 3 & 25.00 & 3 & 12 & 22.22 \\
\hline 4 & 36.36 & 4 & 11 & 4 & 27.27 & 3 & 11 & 4 & 27.27 & 3 & 11 & 30.30 \\
\hline 5 & 36.36 & 4 & 11 & 5 & 30.00 & 3 & 10 & 5 & 44.44 & 4 & 9 & 36.94 \\
\hline 6 & 36.36 & 4 & 11 & 6 & 36.36 & 4 & 11 & 6 & 41.67 & 5 & 12 & 38.13 \\
\hline 7 & 25.00 & 3 & 12 & 7 & 45.45 & 5 & 11 & 7 & 36.36 & 4 & 11 & 35.61 \\
\hline 8 & 36.36 & 4 & 11 & 8 & 30.00 & 3 & 10 & 8 & 45.45 & 5 & 11 & 37.27 \\
\hline 9 & 30.77 & 4 & 13 & 9 & 30.77 & 4 & 13 & 9 & 20.00 & 2 & 10 & 27.18 \\
\hline 10 & 25.00 & 3 & 12 & 10 & 36.36 & 4 & 11 & 10 & 27.27 & 3 & 11 & 29.55 \\
\hline 11 & 15.38 & 2 & 13 & 11 & 7.69 & 1 & 13 & 11 & 18.18 & 2 & 11 & 13.75 \\
\hline 12 & 18.18 & 2 & 11 & 12 & 0.00 & 0 & 10 & 12 & 8.33 & 1 & 12 & 8.84 \\
\hline 13 & 30.00 & 3 & 10 & 13 & 27.27 & 3 & 11 & 13 & 25.00 & 3 & 12 & 27.42 \\
\hline 14 & 16.67 & 2 & 12 & 14 & 8.33 & 1 & 12 & 14 & 11.11 & 1 & 9 & 12.04 \\
\hline 15 & 9.09 & 1 & 11 & 15 & 0.00 & 0 & 11 & 15 & 12.50 & 1 & 8 & 7.20 \\
\hline \multirow[t]{3}{*}{ 2017-18 } & & & & & & & & & & & & 24.57 \\
\hline & & RI & & & & $\mathrm{R} 2$ & & & & RI & & \\
\hline & $\begin{array}{c}\text { Per cent } \\
\text { disease } \\
\text { incidence }\end{array}$ & $\begin{array}{l}\text { No. of } \\
\text { grapevine } \\
\text { leaves } \\
\text { affected }\end{array}$ & $\begin{array}{c}\text { Total no. } \\
\text { of leaves } \\
\text { observed } \\
\text { in a set }\end{array}$ & & $\begin{array}{l}\text { Per cent } \\
\text { disease } \\
\text { incidence }\end{array}$ & $\begin{array}{c}\text { No. of } \\
\text { grapevine } \\
\text { leaves } \\
\text { affected }\end{array}$ & $\begin{array}{c}\text { Total no. of } \\
\text { leaves } \\
\text { observed in a } \\
\text { set }\end{array}$ & & $\begin{array}{c}\text { Per cent } \\
\text { disease } \\
\text { incidence }\end{array}$ & $\begin{array}{l}\text { No. of } \\
\text { grapevine } \\
\text { leaves } \\
\text { affected }\end{array}$ & $\begin{array}{c}\text { Total no. } \\
\text { of leaves } \\
\text { observed } \\
\text { in a set }\end{array}$ & $\begin{array}{c}\text { Mean } \\
\text { PDI }\end{array}$ \\
\hline 1 & 33.33 & 4 & 12 & 1 & 40.00 & 4 & 10 & 1 & 30.00 & 3 & 10 & 34.44 \\
\hline 2 & 9.09 & 1 & 11 & 2 & 8.33 & 1 & 12 & 2 & 16.67 & 2 & 12 & 11.36 \\
\hline
\end{tabular}




\begin{tabular}{|c|c|c|c|c|c|c|c|c|c|c|c|c|}
\hline 3 & 23.08 & 3 & 13 & 3 & 41.67 & 5 & 12 & 3 & 25.00 & 3 & 12 & 29.91 \\
\hline 4 & 8.33 & 1 & 12 & 4 & 18.18 & 2 & 11 & 4 & 0.00 & 0 & 11 & 8.84 \\
\hline 5 & 27.27 & 3 & 11 & 5 & 27.27 & 3 & 11 & 5 & 23.08 & 3 & 13 & 25.87 \\
\hline 6 & 50.00 & 5 & 10 & 6 & 33.33 & 4 & 12 & 6 & 45.45 & 5 & 11 & 42.93 \\
\hline 7 & 8.33 & 1 & 12 & 7 & 45.45 & 5 & 11 & 7 & 33.33 & 4 & 12 & 29.04 \\
\hline 8 & 36.36 & 4 & 11 & 8 & 50.00 & 5 & 10 & 8 & 41.67 & 5 & 12 & 42.68 \\
\hline 9 & 38.46 & 5 & 13 & 9 & 38.46 & 5 & 13 & 9 & 30.00 & 3 & 10 & 35.64 \\
\hline 10 & 25.00 & 3 & 12 & 10 & 23.08 & 3 & 13 & 10 & 18.18 & 2 & 11 & 22.09 \\
\hline 11 & 45.45 & 5 & 11 & 11 & 46.15 & 6 & 13 & 11 & 33.33 & 4 & 12 & 41.65 \\
\hline 12 & 45.45 & 5 & 11 & 12 & 16.67 & 2 & 12 & 12 & 16.67 & 2 & 12 & 26.26 \\
\hline 13 & 30.00 & 3 & 10 & 13 & 27.27 & 3 & 11 & 13 & 27.27 & 3 & 11 & 28.18 \\
\hline 14 & 10.00 & 1 & 10 & 14 & 7.69 & 1 & 13 & 14 & 7.69 & 1 & 13 & 8.46 \\
\hline \multirow[t]{2}{*}{15} & 46.15 & 6 & 13 & 15 & 36.36 & 4 & 11 & 15 & 16.67 & 2 & 12 & 33.06 \\
\hline & & & & & & & & & & & & 28.03 \\
\hline \multirow[t]{2}{*}{ 2018-19 } & & RI & & & & $\mathrm{R} 2$ & & & & RI & & \\
\hline & $\begin{array}{c}\text { Per cent } \\
\text { disease } \\
\text { incidence }\end{array}$ & $\begin{array}{c}\text { No. of } \\
\text { grapevine } \\
\text { leaves } \\
\text { affected }\end{array}$ & $\begin{array}{c}\text { Total no. } \\
\text { of leaves } \\
\text { observed } \\
\text { in a set }\end{array}$ & & $\begin{array}{c}\text { Per cent } \\
\text { disease } \\
\text { incidence }\end{array}$ & $\begin{array}{c}\text { No. of } \\
\text { grapevine } \\
\text { leaves } \\
\text { affected }\end{array}$ & $\begin{array}{l}\text { Total no. of } \\
\text { leaves } \\
\text { observed in a } \\
\text { set }\end{array}$ & & $\begin{array}{c}\text { Per cent } \\
\text { disease } \\
\text { incidence }\end{array}$ & $\begin{array}{c}\text { No. of } \\
\text { grapevine } \\
\text { leaves } \\
\text { affected }\end{array}$ & $\begin{array}{c}\text { Total no. } \\
\text { of leaves } \\
\text { observed } \\
\text { in a set }\end{array}$ & $\begin{array}{c}\text { Mean } \\
\text { PDI }\end{array}$ \\
\hline 1 & 18.18 & 2 & 11 & 1 & 30.00 & 3 & 10 & 1 & 27.27 & 3 & 11 & 25.15 \\
\hline 2 & 27.27 & 3 & 11 & 2 & 8.33 & 1 & 12 & 2 & 25.00 & 3 & 12 & 20.20 \\
\hline 3 & 30.77 & 4 & 13 & 3 & 41.67 & 5 & 12 & 3 & 25.00 & 3 & 12 & 32.48 \\
\hline 4 & 0.00 & 0 & 12 & 4 & 9.09 & 1 & 11 & 4 & 11.11 & 1 & 9 & 6.73 \\
\hline 5 & 45.45 & 5 & 11 & 5 & 36.36 & 4 & 11 & 5 & 27.27 & 3 & 11 & 36.36 \\
\hline 6 & 30.00 & 3 & 10 & 6 & 16.67 & 2 & 12 & 6 & 25.00 & 3 & 12 & 23.89 \\
\hline 7 & 16.67 & 2 & 12 & 7 & 27.27 & 3 & 11 & 7 & 18.18 & 2 & 11 & 20.71 \\
\hline 8 & 27.27 & 3 & 11 & 8 & 40.00 & 4 & 10 & 8 & 41.67 & 5 & 12 & 36.31 \\
\hline 9 & 23.08 & 3 & 13 & 9 & 0.00 & 0 & 13 & 9 & 20.00 & 2 & 10 & 14.36 \\
\hline 10 & 25.00 & 3 & 12 & 10 & 15.38 & 2 & 13 & 10 & 18.18 & 2 & 11 & 19.52 \\
\hline
\end{tabular}




\begin{tabular}{|c|c|c|c|c|c|c|c|c|c|c|c|c|}
\hline 11 & 15.38 & 2 & 13 & 11 & 15.38 & 2 & 13 & 11 & 7.69 & 1 & 13 & 12.82 \\
\hline 12 & 9.09 & 1 & 11 & 12 & 16.67 & 2 & 12 & 12 & 0.00 & 0 & 12 & 8.59 \\
\hline 13 & 0.00 & 0 & 10 & 13 & 18.18 & 2 & 11 & 13 & 23.08 & 3 & 13 & 13.75 \\
\hline 14 & 30.00 & 3 & 10 & 14 & 30.77 & 4 & 13 & 14 & 50.00 & 5 & 10 & 36.92 \\
\hline \multirow[t]{2}{*}{15} & 30.77 & 4 & 13 & 15 & 27.27 & 3 & 11 & 15 & 25.00 & 3 & 12 & 27.68 \\
\hline & & & & & & & & & & & & 22.37 \\
\hline \multirow[t]{2}{*}{ 2019-20 } & & RI & & & & $\mathrm{R} 2$ & & & & RI & & \\
\hline & $\begin{array}{c}\text { Per cent } \\
\text { disease } \\
\text { incidence }\end{array}$ & $\begin{array}{l}\text { No. of } \\
\text { grapevine } \\
\text { leaves } \\
\text { affected }\end{array}$ & $\begin{array}{c}\text { Total no. } \\
\text { of leaves } \\
\text { observed } \\
\text { in a set }\end{array}$ & & $\begin{array}{l}\text { Per cent } \\
\text { disease } \\
\text { incidence }\end{array}$ & $\begin{array}{l}\text { No. of } \\
\text { grapevine } \\
\text { leaves } \\
\text { affected }\end{array}$ & $\begin{array}{c}\text { Total no. of } \\
\text { leaves } \\
\text { observed in a } \\
\text { set }\end{array}$ & & $\begin{array}{l}\text { Per cent } \\
\text { disease } \\
\text { incidence }\end{array}$ & $\begin{array}{c}\text { No. of } \\
\text { grapevine } \\
\text { leaves } \\
\text { affected }\end{array}$ & $\begin{array}{c}\text { Total no. } \\
\text { of leaves } \\
\text { observed } \\
\text { in a set }\end{array}$ & $\begin{array}{c}\text { Mean } \\
\text { PDI }\end{array}$ \\
\hline 1 & 27.27 & 3 & 11 & 1 & 30.00 & 3 & 10 & 1 & 44.44 & 4 & 9 & 33.91 \\
\hline 2 & 30.00 & 3 & 10 & 2 & 8.33 & 1 & 12 & 2 & 25.00 & 3 & 12 & 21.11 \\
\hline 3 & 0.00 & 0 & 12 & 3 & 8.33 & 1 & 12 & 3 & 8.33 & 1 & 12 & 5.56 \\
\hline 4 & 27.27 & 3 & 11 & 4 & 40.00 & 4 & 10 & 4 & 27.27 & 3 & 11 & 31.52 \\
\hline 5 & 36.36 & 4 & 11 & 5 & 20.00 & 2 & 10 & 5 & 18.18 & 2 & 11 & 24.85 \\
\hline 6 & 0.00 & 0 & 10 & 6 & 0.00 & 0 & 11 & 6 & 30.00 & 3 & 10 & 10.00 \\
\hline 7 & 27.27 & 3 & 11 & 7 & 40.00 & 4 & 10 & 7 & 36.36 & 4 & 11 & 34.55 \\
\hline 8 & 16.67 & 2 & 12 & 8 & 27.27 & 3 & 11 & 8 & 16.67 & 2 & 12 & 20.20 \\
\hline 9 & 23.08 & 3 & 13 & 9 & 23.08 & 3 & 13 & 9 & 20.00 & 2 & 10 & 22.05 \\
\hline 10 & 33.33 & 3 & 9 & 10 & 30.77 & 4 & 13 & 10 & 45.45 & 5 & 11 & 36.52 \\
\hline 11 & 0.00 & 0 & 12 & 11 & 8.33 & 1 & 12 & 11 & 7.69 & 1 & 13 & 5.34 \\
\hline 12 & 18.18 & 2 & 11 & 12 & 8.33 & 1 & 12 & 12 & 16.67 & 2 & 12 & 14.39 \\
\hline 13 & 0.00 & 0 & 10 & 13 & 18.18 & 2 & 11 & 13 & 7.69 & 1 & 13 & 8.62 \\
\hline 14 & 30.00 & 3 & 10 & 14 & 25.00 & 3 & 12 & 14 & 30.00 & 3 & 10 & 28.33 \\
\hline \multirow[t]{2}{*}{15} & 16.67 & 2 & 12 & 15 & 27.27 & 3 & 11 & 15 & 8.33 & 1 & 12 & 17.42 \\
\hline & & & & & & & & & & & & 20.96 \\
\hline
\end{tabular}


Appendix.1 MATLAB Program to find the simulation using the regression equations (5) and (6)

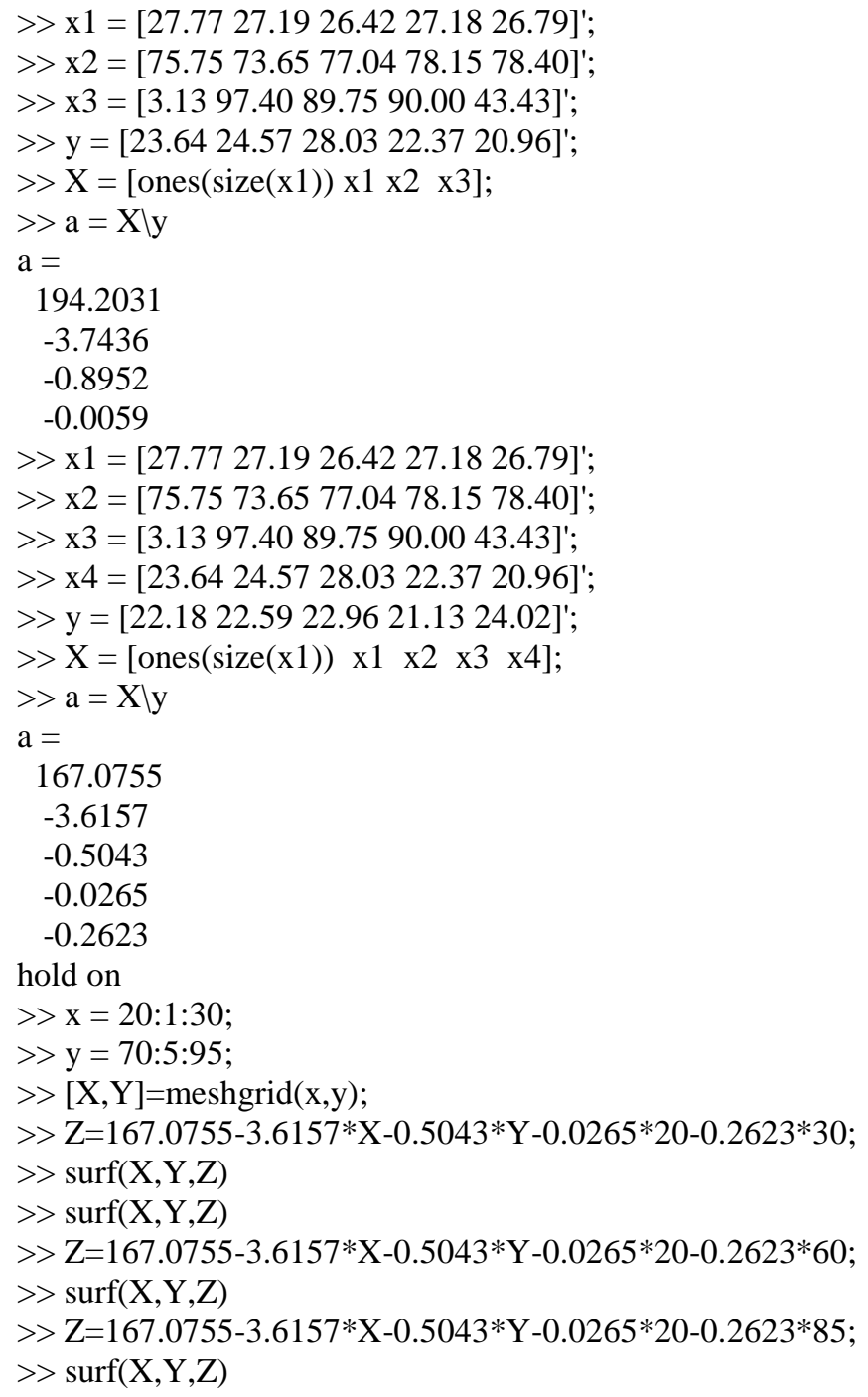


Fig.1 Structural diagram of the information system for determining the probability of reduction in the yield of grapes.

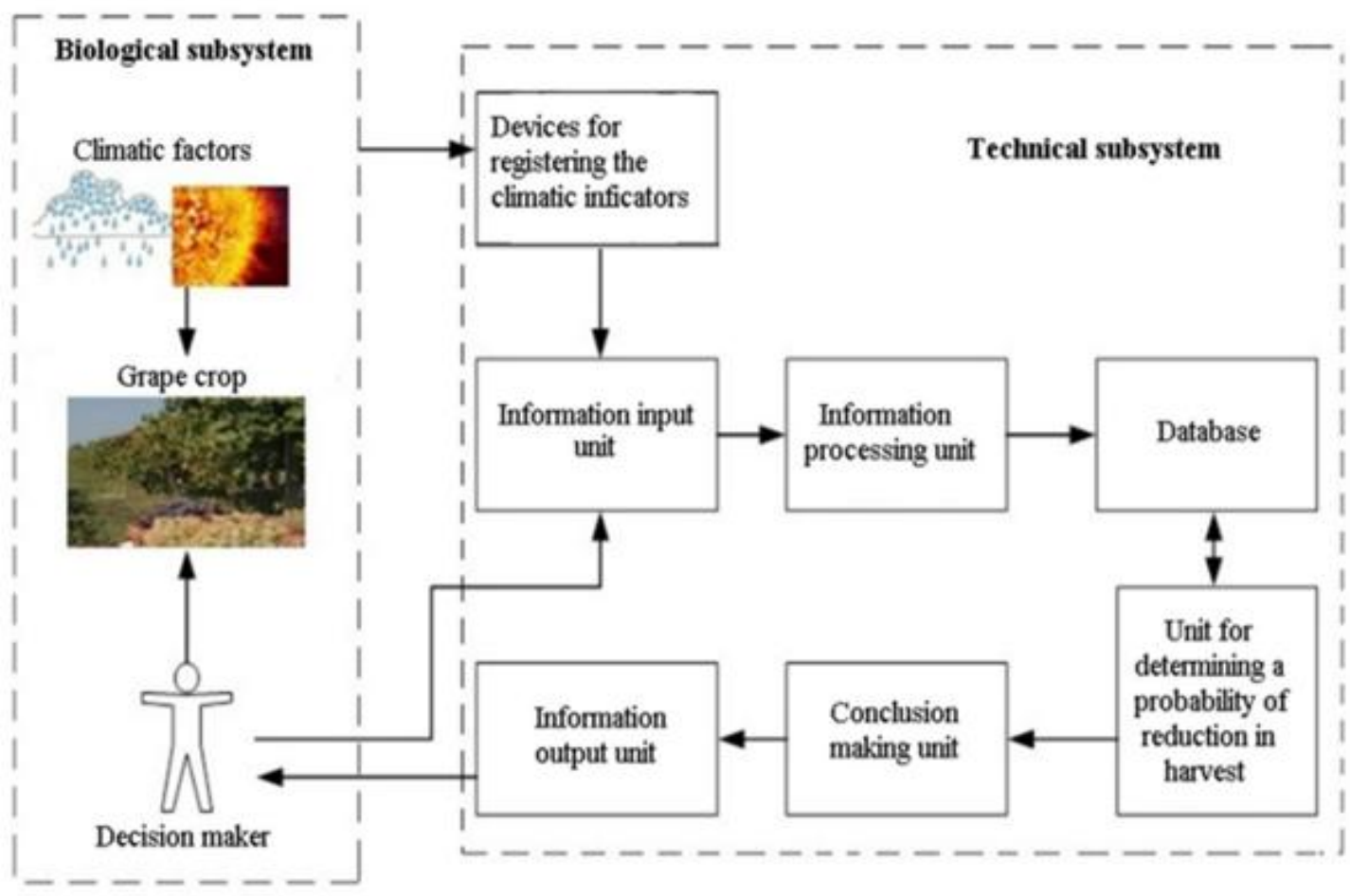

Fig.2a Relation between temperature, Relative humidity and grape yield for the value of $\mathrm{PDI}=30 \%$ and Rainfall $=20(\mathrm{~mm})$.

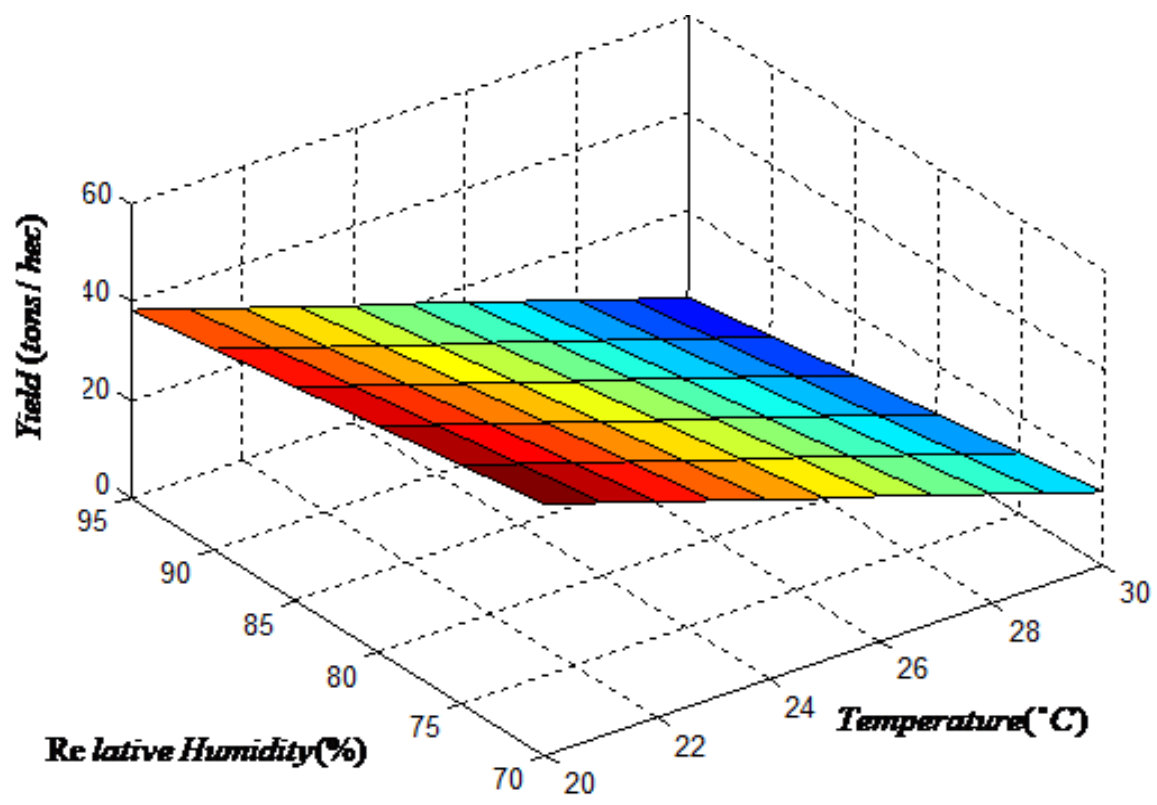


Fig.2b Relation between temperature, Relative humidity and grape yield for the value of $\mathrm{PDI}=60 \%$ and Rainfall $=20(\mathrm{~mm})$.

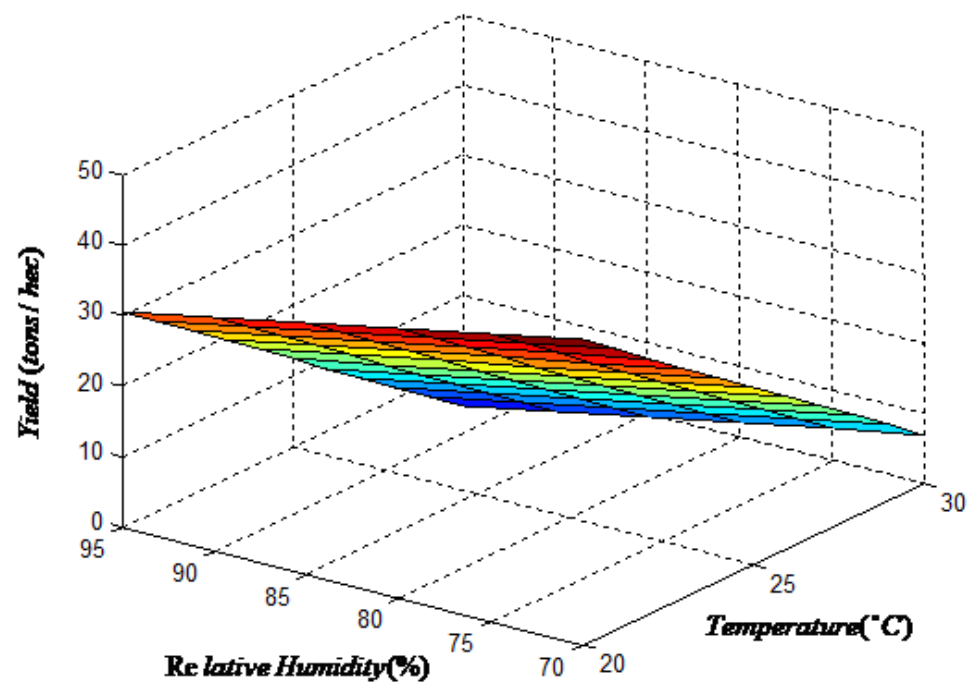

Fig.2c Relation between temperature, Relative humidity and grape yield for the value of $\mathrm{PDI}=85 \%$ and Rainfall $=20(\mathrm{~mm})$.

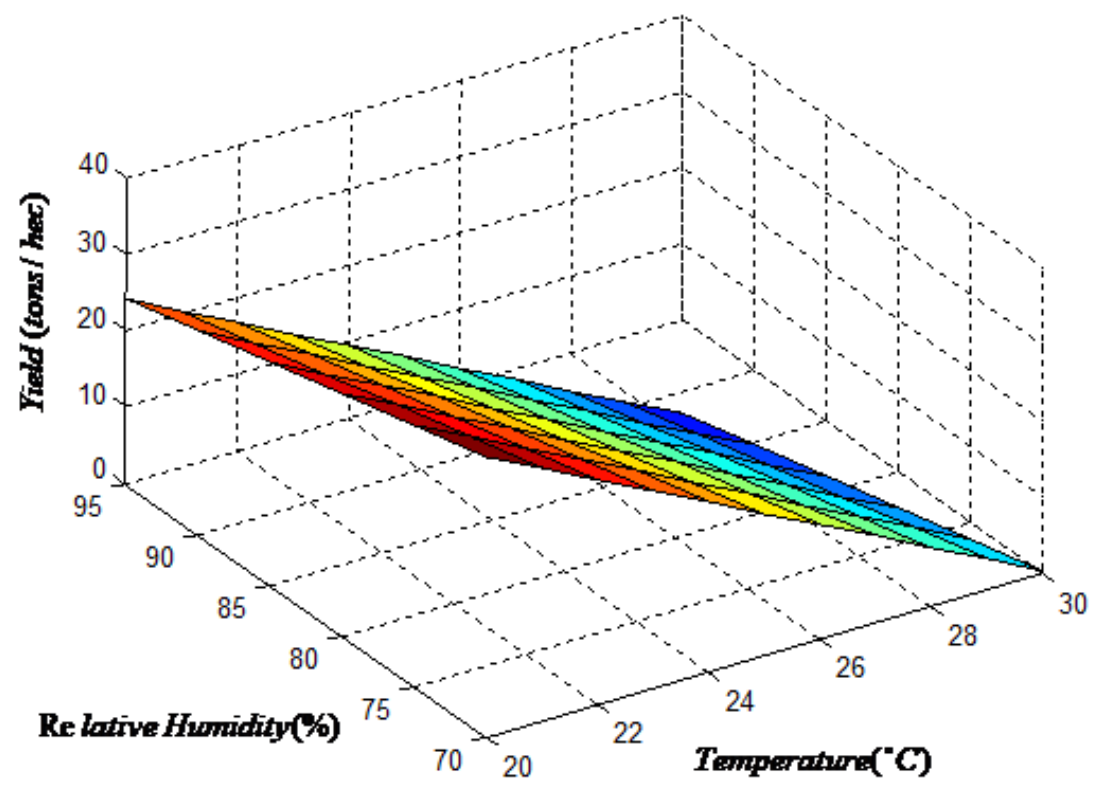


Fig.3 Relation between temperature and needed leaf wetness duration for infection.

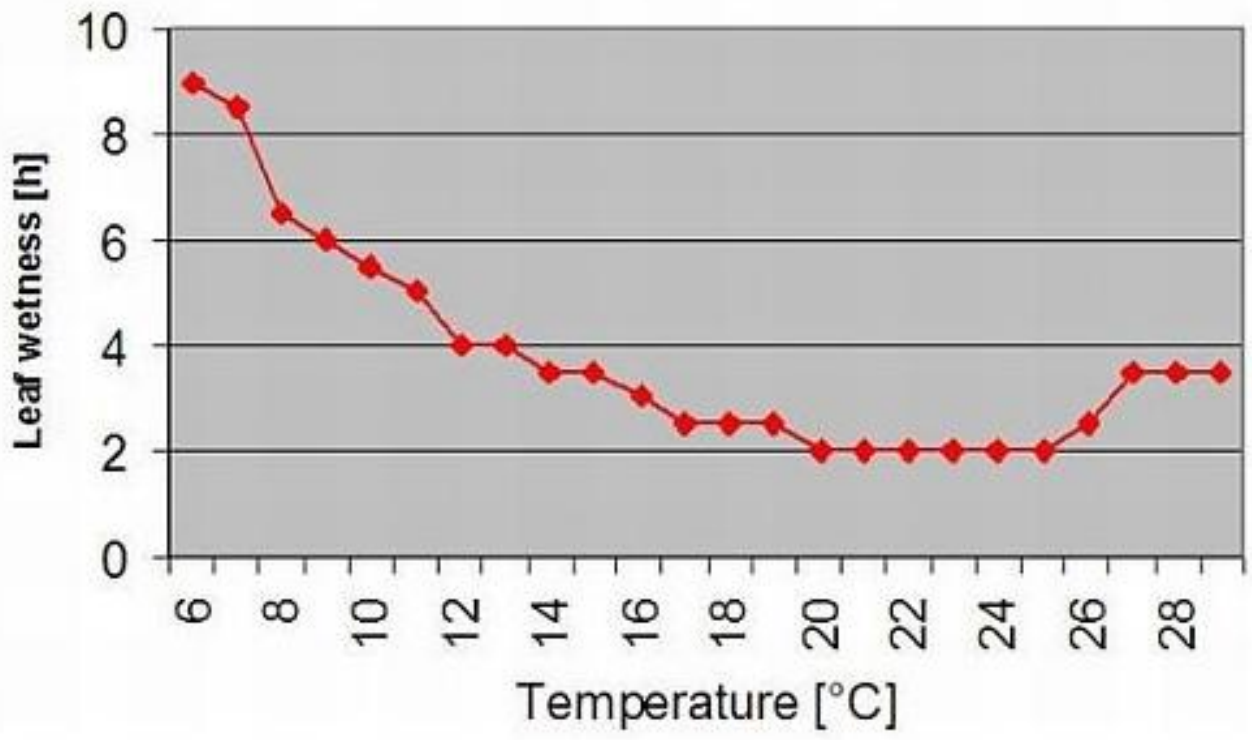

Fig.4 Relation between temperature and sporulation progress.

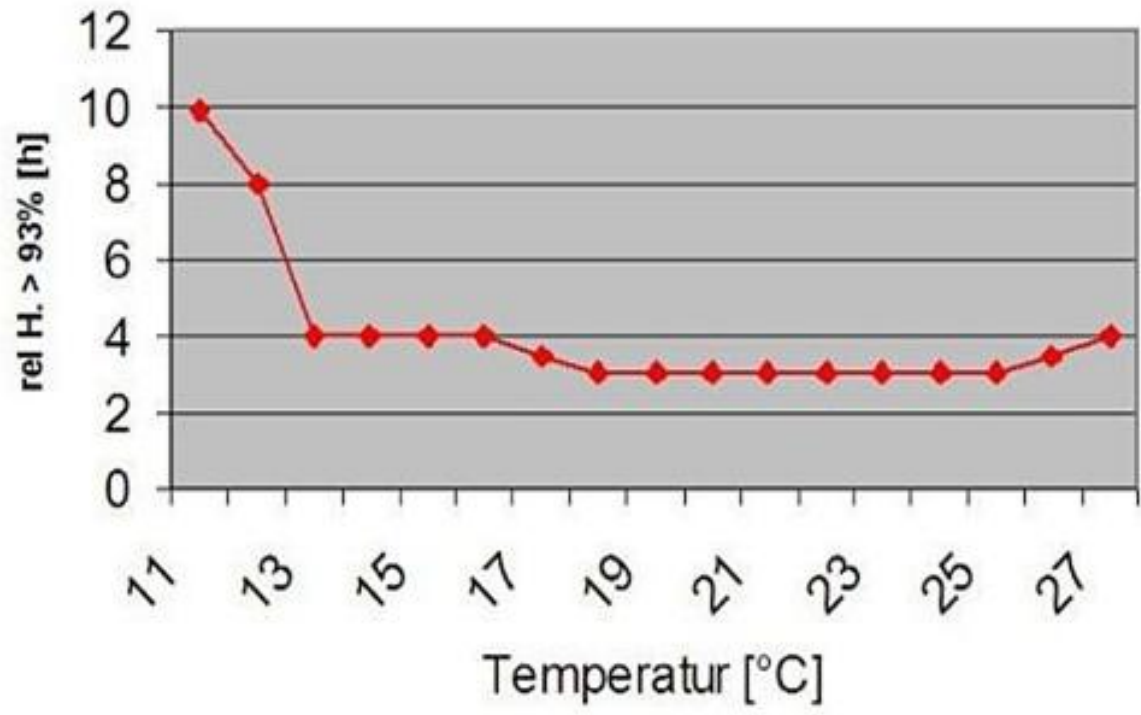


Fig.5a Relation between temperature, Rain fall and Percentage disease incidence for fixed value of $\mathrm{RH}=95 \%$.

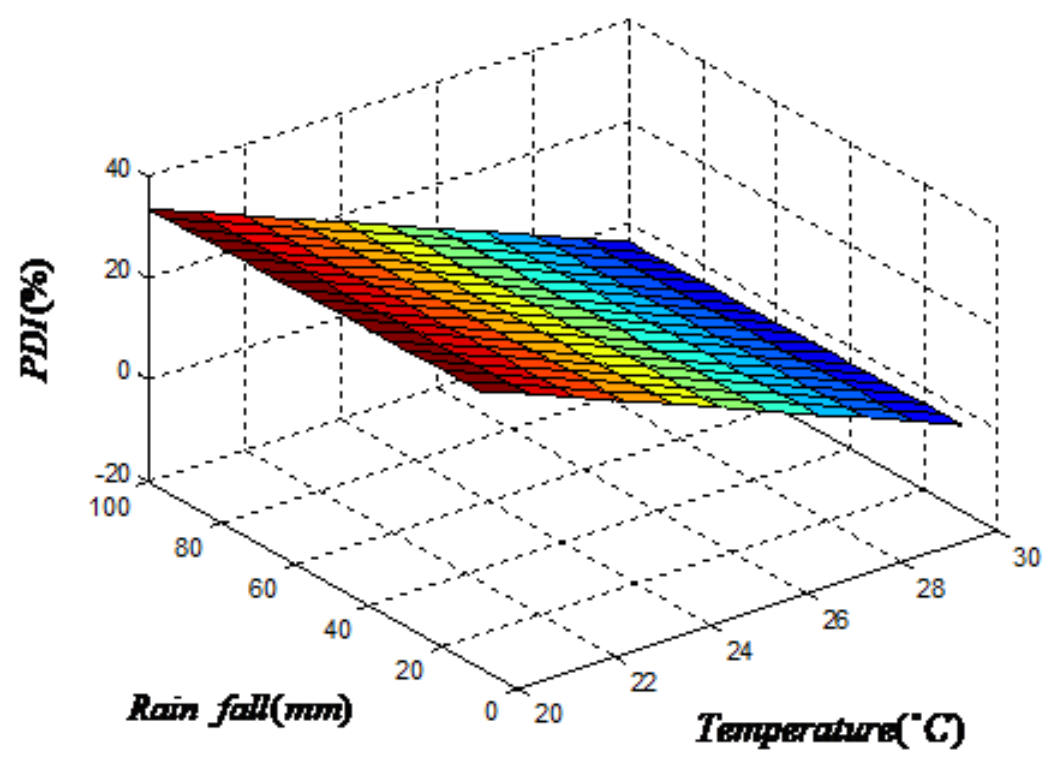

Fig.5b Relation between temperature, Rain fall and Percentage disease incidence for fixed value of $\mathrm{RH}=75 \%$.

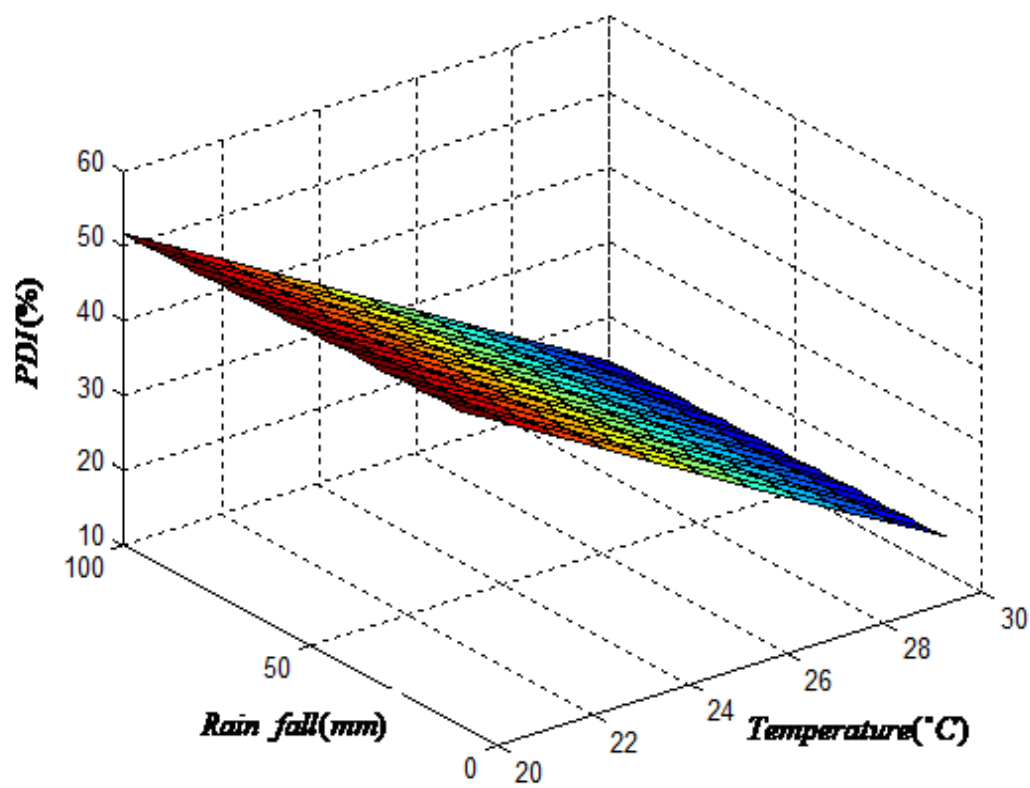


Fig.6a The temperature for maximum and minimum versus calendar months for five years meteorological data in grape.

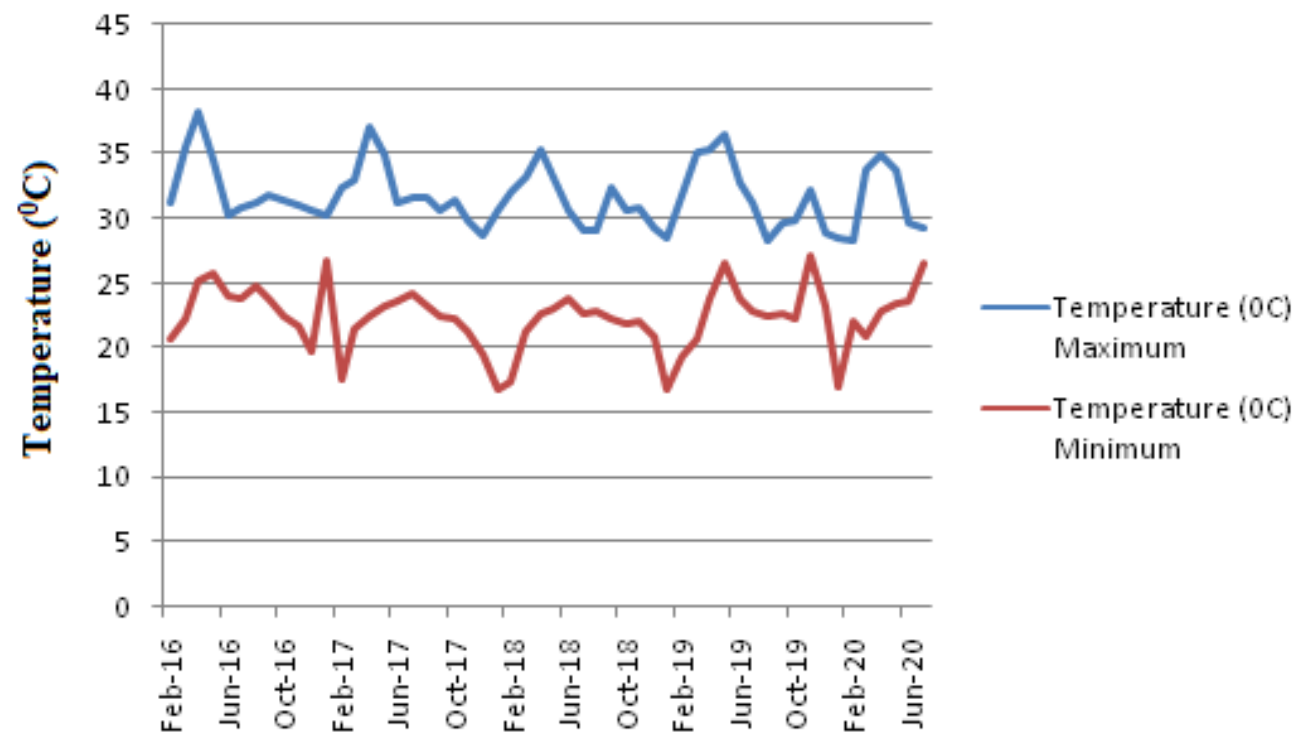

\section{Calender months}

Fig.6b The meteorological data versus calendar month for five years climatic parameters RH (\%) and RF (mm).

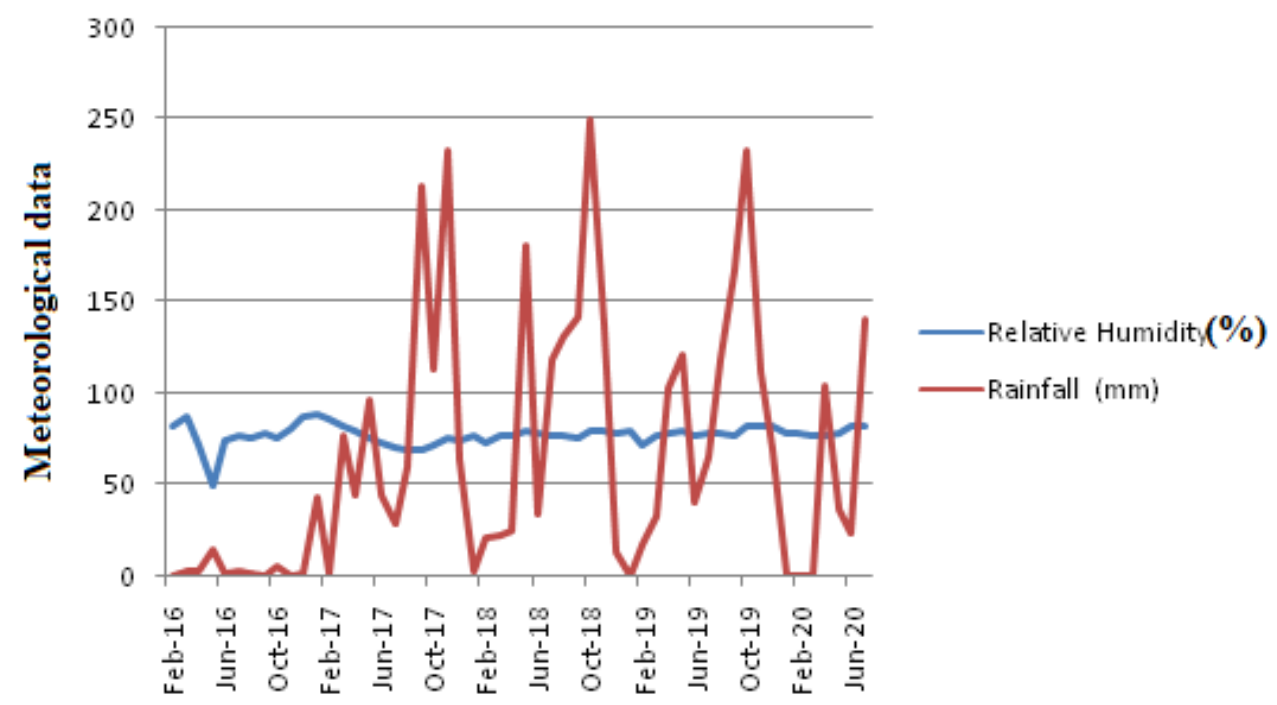

Calender months 
Fig.7 The weather condition for relative humidity (\%) versus calendar months for five years meteorological data in grape.

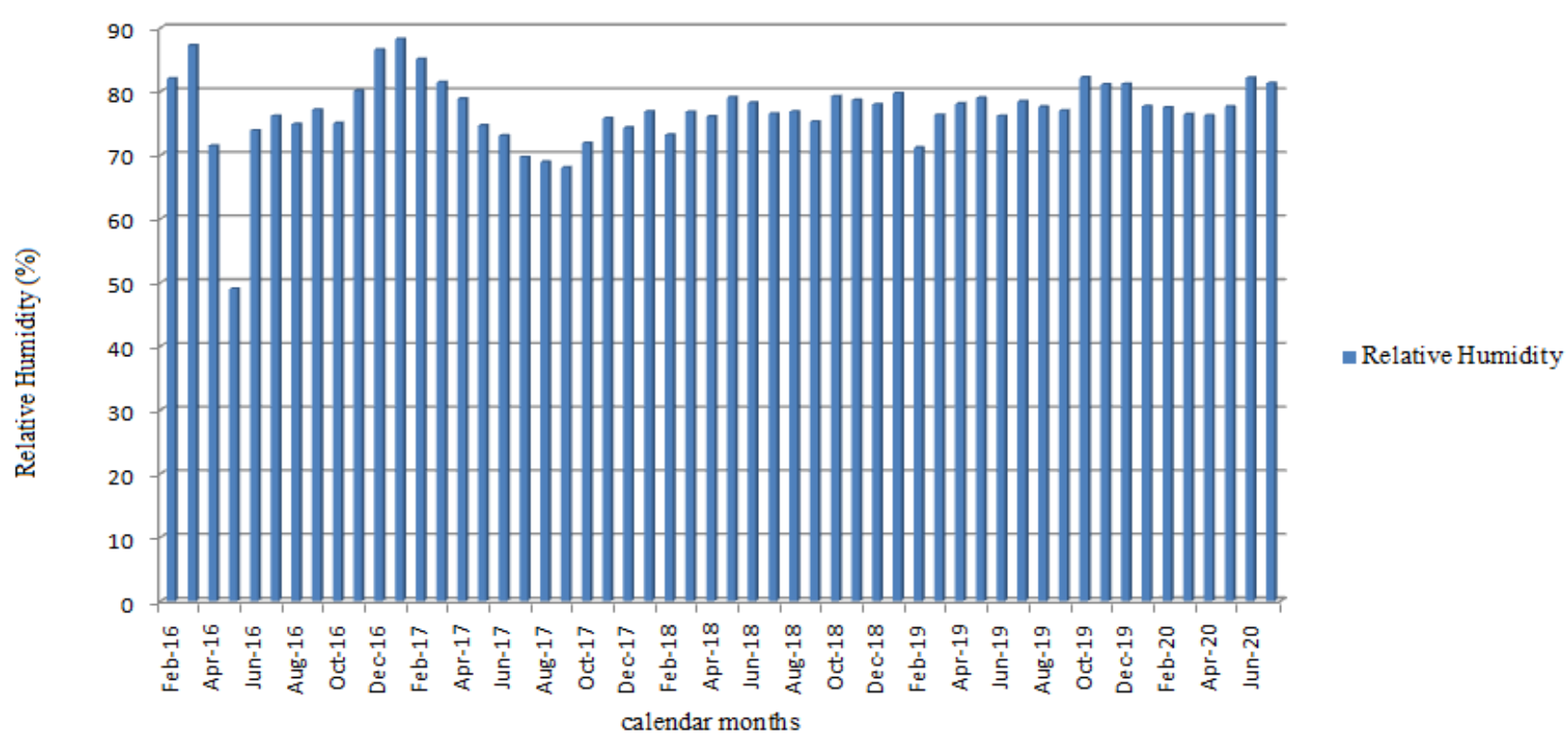

Fig.8 The weather condition for rainfall $(\mathrm{mm})$ versus calendar months for five years meteorological data in grape.

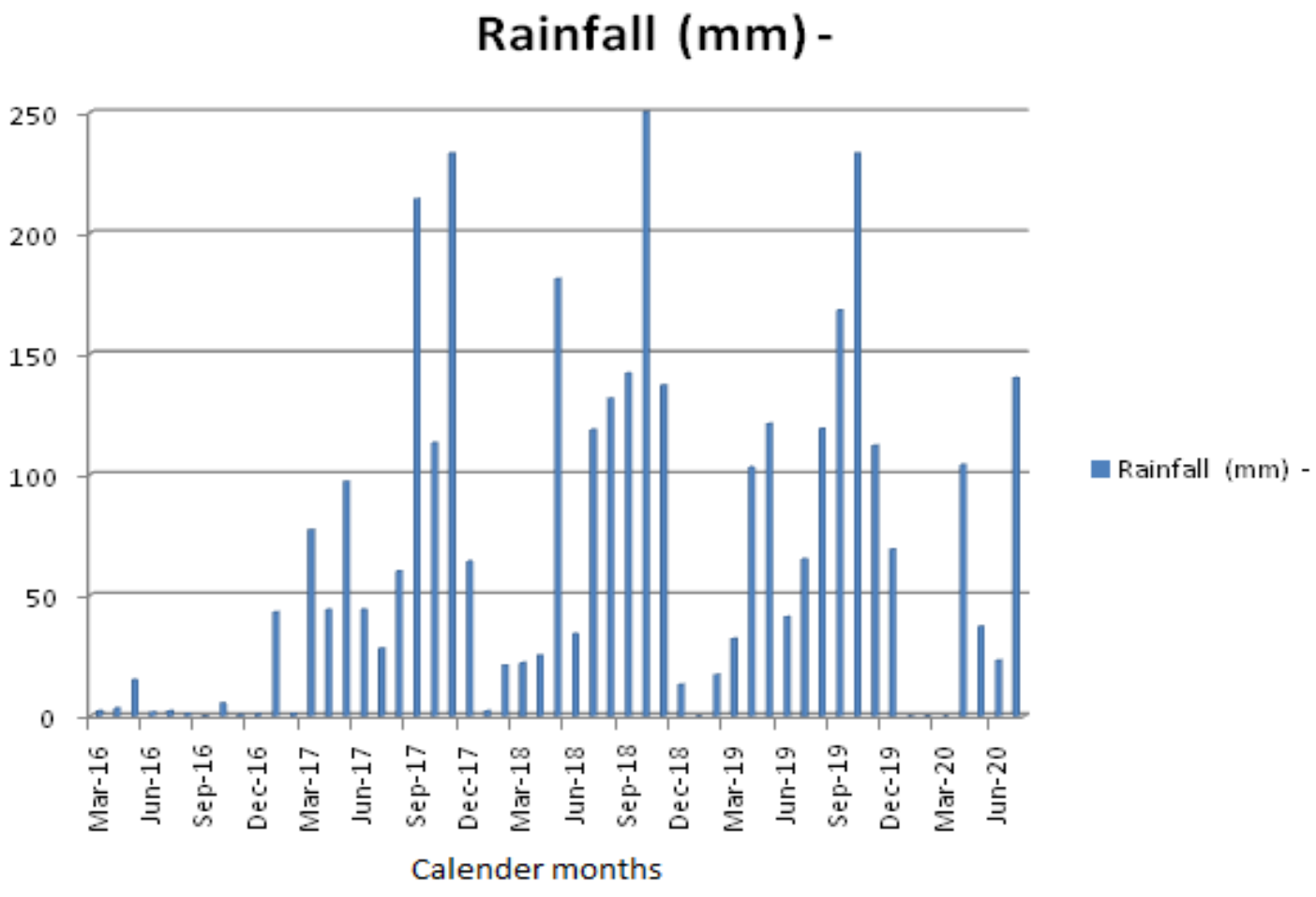


Fig.9 Standard area diagram for assessment of downy mildew disease of grape.

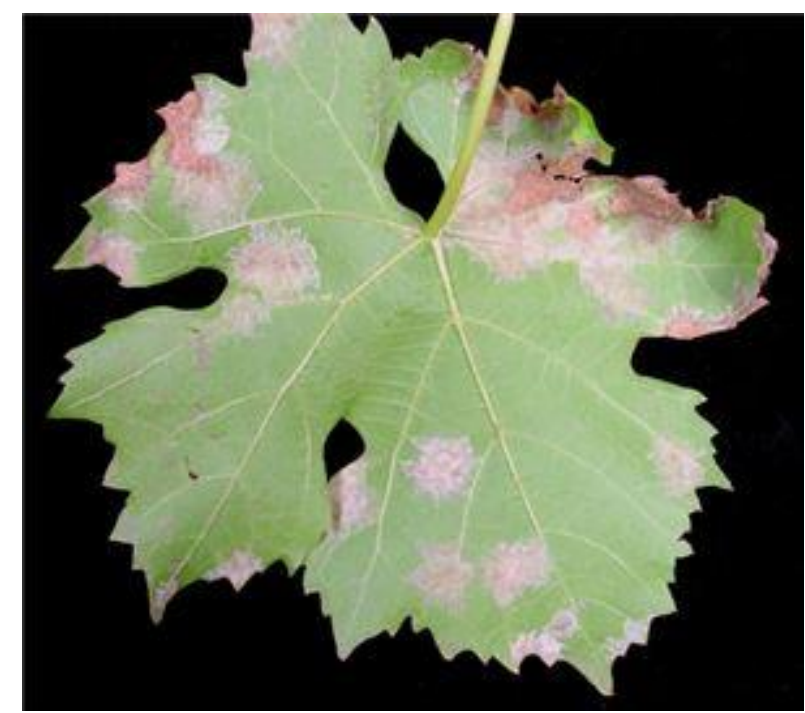

We identified three major indicators such as Temperature, Relative humidity and Rainfall. They make it possible to estimate the probability of reduction in the yield of grapes at Theni surrounding like Kumbum, K.K Patty, Odaipatty, Suruli patty and Chinnamanur, which is calculated under GRS weather conditions. We obtained a mathematical model for evaluating the reduction in grape yield by using the multiple regressions method.

This proposed model could be used to make a decision on in order to increase the yield of grapes. The developed model is reliable and adequate with the actual data while all predictors of the model, in accordance with the RMSE test, to identify the prediction on reducing the yield of grape. Also, we can find the PDI model as a function of temperature, relative humidity, rainfall and yield.

The developed model through this paper will be helpful in grape farmers in Theni district of Tamil Nadu. These model are applied to use enhance or alter the natural growth and fruit development pattern or it can be used to control (or) prevent pest and disease outbreak in grapevines.

\section{Acknowledgement}

This work was supported by Science and Engineering Research Board under MATRICS (SERB - No.: MTR/2019/001221). The Authors are also express their gratitude to the Vice Chancellor Dr. N. Kumar and Director of Research Dr.K.S.Subramanian, Tamil Nadu Agricultural University, Coimbatore, Dr. B. Shridar, The Dean, Agricultural Engineering College and Research Institute, TNAU, Coimbatore, Tamil Nadu, India for their constant support and encouragement.

\section{References}

Bonfigli. M, Godoy. E,Reinheimer.M.A, Scenna.N.J, Comparison between conventional and ultrasound-assisted techniques for extraction of anthocyanins from grape pomace. Experimental results and mathematical modelling, J. Food.Engg. (2020)

Bournier, A. Grape insects. Annu. Rev. Entomol. 22 1977 355-376.

Caffi. T and Rossi.V, Evaluation of a Warning System for Controlling Primary Infections of Grapevine Downy 
Mildew, Plant Disease, June 2010. doi:10.1094/ PDIS-94-6-0709

Fengjiao fan, Jianping li, Guominggao, Chenxi ma, Mathematical model application based on statistics in the evaluation analysis of grape wine quality, $978-1-4673-8266-3 / 15 / \$ 31.00$ C1015 IEEE.2015

Flaherty, D.L.; Jensen, F.; Kasimatis, A.; Kido, H.; Moller, W. Grape Pest Management; Agricultural Sciences Publications, University of California:Berkeley, CA, USA, 1981.

Hans Peter Fischer, Mathematical Modeling of Complex Biological Systems, Alcohol Res Health. 31(1) 2008 49-59.

MATLAB 6.1. Natick, MA: The Math Works Inc.; 2000. sss $<w w w . s c i l a b e n t e r p r i s e s . c o m>$.
Ragusa, S.; Tsolakis, H. (Eds.) La Difesa Della Vite Dagli Artropodi Dannosi; UniversitàdegliStudi di Palermo: Palermo, Italy, 2006.

Tanmay A. Wagh, R. M. Samant, Sharvil V. Gujarathi, Snehal B. Gaikwad, Grapes Leaf Disease Detection using Convolutional Neural Network, Int. J. of Comp. Appl., (0975 - 8887) Volume 178 - No. 20, June 2019.

Vittorio Faluomi,IacopoBorsi, Vinesym: an integrated vine and grapevine mathematical model for vegetative development and production quality forecast, BIO Web of Conferences 13, 02005 (2019). https://doi.org/10.1051/bioconf/201913 02005

\section{How to cite this article:}

Eswari, A. 2021. Weather Based Yield Prediction and PDI Model for Grape Production Quality Forecast in Tamil Nadu using Mathematical Modelling. Int.J.Curr.Microbiol.App.Sci. 10(04): 653-670. doi: https://doi.org/10.20546/ijcmas.2021.1004.066 